\title{
A numerical model for run-up of breaking waves.
}

\author{
by \\ H. JOHNSGARD \\ Department of Mathematics University of Oslo \\ P.O.Box 1053 Blindern Oslo Norway
}

29th January 1998

\begin{abstract}
In the present paper the numerical method for three dimensional runup, given in Johnsgard and Pedersen, [4], is extended to include wave breaking. In the fundamental problem of run-up of a uniform bore we compare our model to analytical solutions from the literature. The numerical solutions converges, but very slowly. This is not due to the numerical model, but rather to the structure of the solutions themself. Numerical results for two realistic, but simplified Tsunami cases are also presented. In the first case we make two-dimensional simulations concerning the run-up of a Tsunami in Portugal, in the second case we study the three dimensional wave pattern generated after a slide in Tafjord, Norway, in 1931. A discussion of different aspects of the model is summarized at the end of the paper.
\end{abstract}

\section{Introduction}

The study of wave breaking during run-up is important for two reasons. First, it it occurs in many practical situations, and influences both run-up heights and the destructive potential of the waves. Second, wave breaking may be hidden in numerical solutions for shallow water equations. Hopefully the reader of this paper will gain improved understanding of when such breaking may occur, and how to identify it.

A full description of wave breaking is a very complicated problem, and the kind of turbulence modeling that is needed, is still not available. A common approach, that is valid in the long wave limit, is to view the bore that is formated during breaking as a discontinuity. The key feature for this approach is that no mass and momentum are lost during breaking, and methods used for acoustic shock waves may be adapted. Such methods may be based on a patching of fluxes for mass and momentum at the bore discontinuity. A more sophisticated use of analytical expressions is made in Gudonovs method, where the surface profile is approximated by a piecewise constant function, and the solution on an advancing time step is found after solving a series of Riemann problems. A large family of techniques have been developed from this basis. A simpler approach is to include some kind of artificial diffusion, either implicitly inherited in the 
numerical scheme, or stated explicitly. For refined grids and vanishing artificial diffusion all these methods converge towards the same limit, as long as care is taken so that mass and momentum are conserved.

In the present paper I generalize the method developed by Johnsgard and Pedersen [4], to include wave breaking. I am not focusing on the shock treatment itself, but rather on the processes involved when shocks are interacting with moving shorelines. Hence the simplest possible treatment of the bore has been selected: an artificial diffusion stated directly in the numerical scheme. We notice that the slow convergence that is reported in this paper is not due to this particular selection of shock treatment.

Hibberd and Peregrine [3], made a related study of bore tun-up as in the present paper. Two new features are added here: the Lagrangian description, that makes the run-up calculations more accurate, and the capacity of solving three dimensional problems.

Classical analytical theory, that is discussed in section 3 , shows an extremely fast bore development near the free tip, and the outcome of this process has substantial influence on the run-up heights. This gives rise to two fundamental problems. First, extremely dense grid is needed to get converged results. Second, since real world bores have finite length, the bore development near the shore may not be physically relevant. A discussion of these questions, also including a bottom drag, will be given in this paper.

The numerical simulation of two simplified, but realistic Tsunami cases has also been included. The coastal and bottom topography has been selected simple enough to make it possible to identify the different physical processes that are involved, and see how they are handled.

\section{The model.}

Gravity waves in inviscid and incompressible fluids are considered. Typical lengths of the waves and scales for bottom variations are sufficiently long for a hydrostatic pressure distribution to apply. The governing equations are the Airy equations, which forms a fully nonlinear, non-dispersive wave model (see for instance Peregrine [6]). Lagrangian coordinates are introduced to enable a tracking of moving shorelines. A simple model of slide events is included into the model through a time dependent bottom topography.

\subsection{Governing equations.}

Following Johnsgard and Pedersen, [4], we now introduce Lagrangian enumeration co-ordinates $a$ and $b$, marking vertical columns of water. Since the label coordinate system may be curvilinear the computional domain in the $a, b$ plane will be a fixed rectangle. The continuity equation is

$$
H \frac{\partial(x, y)}{\partial(a, b)}=V(a, b)
$$

where $H \equiv h+\eta$ is the total water depth and $V=\left.H(a, b, 0) \frac{\partial(x, y)}{\partial(a, b)}\right|_{t=0}$ has interpretation of volume density per. area in the $(a, b)$ plane. The $x$-component 
of the momentum equation may be written

$$
\frac{\partial^{2} x}{\partial t^{2}}=-\frac{H}{V} \frac{\partial(\eta, y)}{\partial(a, b)}+\frac{\gamma}{H_{0}} \frac{\partial}{\partial a}\left(\frac{H^{q+1}}{H_{0}} \frac{\partial u}{\partial a}\right)+\frac{\gamma}{H_{0}} \frac{\partial}{\partial b}\left(\frac{H^{q+1}}{H_{0}} \frac{\partial u}{\partial b}\right)-\frac{C_{D}|\vec{v}| u}{H_{n}},
$$

where $H_{n}=\max (H, n)$. Selecting $n>0$ will remove the unphysical singularity in the expression for bottom drag. The $y$ - component takes a similar form.

Introducing a horizontal and a vertical length scale, $l_{h}$ and $l_{v}$ respectively, we find that the governing equations may be written in nondimensional form simply by replacing $g$ with 1 and $C_{D}$ and $\gamma$ with $\hat{C}_{D}=\frac{l_{h}}{l_{v}} C_{D}$ and $\hat{\gamma}=\frac{l_{h}}{l_{v}} \gamma$

\subsection{Numerical equations.}

We denote a discrete approximation to a function $f$, at $a=i \Delta a, b=j \Delta b$ and $t=p \Delta t$, by $f=f_{i, j}^{(p)}$. Following Johnsgard and Pedersen, [4], we now discretize the governing equation on an Arakawa B grid, where the primary unknowns are

$$
\eta_{i, j}^{(p)}, H_{i, j}^{(p)}, x_{i+\frac{1}{2}, j+\frac{1}{2}}^{(p)}, y_{i+\frac{1}{2}, j+\frac{1}{2}}^{(p)} .
$$

The numerical equations takes the same form as in Johnsgard and Pedersen [4], except for the inclusion of new terms in the momentum equations, the $x-$ component reads

$$
\begin{array}{r}
{\left[\left[\delta_{t}^{2} x=-g \frac{{\overline{\bar{H}^{a}}}^{b}}{{\overline{\bar{V}_{0}}}^{b}}\left(\overline{\delta_{a} \eta \delta_{b} y}{ }^{b}-{\overline{\delta_{b} \eta \delta_{a} y}}^{a}\right)\right]^{(p)}+\right.} \\
\left.\frac{\Delta t \beta}{\bar{H}_{0}} \delta_{a}\left(\frac{H^{q+1}}{H_{0}} \delta_{a} \delta_{t} x^{(p+1)}\right)+\frac{\Delta t \beta}{\bar{H}_{0}} \delta_{b}\left(\frac{H^{q+1}}{H_{0}} \delta_{b} \delta_{t} x^{(p+1)}\right)-\frac{C_{D}\left|\vec{v}^{(p)}\right| u^{(p+1)}}{H_{n}}\right]_{i+\frac{1}{2}, j+\frac{1}{2}}
\end{array}
$$

Here $\delta_{a}$ and $\bar{f}^{(a)}$ denote a central difference and average operators, and $\gamma=\beta \Delta t$ This closed set of equations for $\delta_{t} x$ is solved with a split step method. The $y-$ component is treated similarly.

We notice that the strength of the diffusion term is reduced as the grid is refined, while the number of grid points within a bore will be held at a fixed level. If $\beta$ is to large the bore becomes to wide, if $\beta$ is to small the numerical solution becomes contaminated by grid noise. Care has been taken so that the optimal choice has been selected for each case.

\section{Run-up of a bore on a uniform slope.}

The numerical method by Johnsgard and Pedersen, [4], that forms the basis for this model, has been extensively tested. I still found it necessary to include a test in the present work where numerical and analytical solutions concerning bore interacting with shorelines were compared. A suitable analytical theory is then the one concerning run-up of uniform bores on uniform slopes. This theory, that was developed in the early sixties, is based on the present governing equations, although we have the possibility to include bottom drag. A short review of this theory will be given, then we turn to the presentation and discussion of the numerical solution. 


\subsection{Bore run-up, existing theory.}

The bore run-up may be divided into several phases:

- On the surf period the bore propagates from the edge of the sloping region to the initial shoreline. A differential equation for the bore velocity as function of the layer thickness in front of the shock was found by Keller et al. [5], by combinations of characteristic expressions behind the bore and bore conditions. The main feature of the solution of this equation is that the bore vanishes as it approaches the shoreline, while the bore strength relative to the layer thickness in front of the shock goes to infinity. There is an almost explosively fast bore development near the shore. For instance, for an initally bore-height of 0.25 times the layer thickness in front of the bore, we find that the particle velocity behind the bore increase from 1.325 to 1.659 during the last $1 / 5000$ fraction of the slope. A good numerical representation of this phenomenon requires extremely dense grid. As we shall see, the run-up heights depend crucially upon the outcome of this process. Off course the physical relevancy of this is highly questionable.

- The next phase is the bore run-up. Analytical results for this process are given by Shen and Meyer [7]. They transformed the governing equations to a linear set by using canonical variables, and showed that the shoreline particle is insensitive to other wave motions. Its position is a quadratic function of time, and the run-up height is given by $R=u_{0}^{2} / g$ where $u_{0}$ is the velocity behind the bore as the bore arrives the shore-line. The layer thickness near the free tip is extremely thin, given by $H=\left(x-x_{s}\right)^{2} / 9 g t^{2}$, where $t$ denotes the time after the bore has arrived at the shoreline.

- The theory developed by Shen and Meyer [7] also predicts the generation of a landward facing bore during back-wash. An almost stationary bore is formed. The effect of this bore is to bring the rapid fluid particles during backwash to nearly rest, and the bore diminishes when the free tip has reached the bore. The water level near the shore will be twice the height of the incident bore, the reflected wave that now has been formed will break, and a seaward facing bore, propagating seawards, with low, gentle waves behind it, will be formed.

\subsection{Model set-up.}

The selected bottom profile is a linear slope in the near-shore region connected to a deep, flat bottom. The equations are put into nondimensional form by selecting the slope length and the depth in the flat region before the bore arrives as horizontal and vertical length scale respectively. A uniform bore with height 0.25 and initial position 1.1 is now incident on the slope, see figure $1 \mathrm{a}$ ). As in Johnsgard and Pedersen ??, we employ a variable initial grid, where the grid size is proportional to $\sqrt{h}$.

Figure 1 shows that the phases of the bore run-up predicted by the analytical theory is reproduced, at least qualitatively. Figure 2 a) shows the shoreline 
elevation for different grids, and the corresponding analytical solution by Meyer. The slow convergence is due to the heavy costs of resolving the inner part of the slope, where the bore develops fast, and are not due to the particular choice of shock treatment. The fluid layer near the free tip is extremely thin. If we redefine the inundation length to be the position where the layer thickness is, say, $10 \%$ of the initial shock height, we find much better convergence, figure 2 b).

An improved physical description of the thin fluid film is achieved by introducing bottom drag into the model. Figure $2 \mathrm{c}$ ) and $2 \mathrm{~d}$ ) shows results for two

different values for $\hat{C}_{D}$. The correct value of $\hat{C}_{D}$ depends on the ratio between the horizontal and vertical scales, for $C_{D}=0.001$ the two cases correspond to $l_{h} / l_{v}=10$ and $l_{h} / l_{v}=100$ respectively. We note that for the first case we have convergence towards roughly the same limit as for the case with $\hat{C}_{D}=0$ and a redefined inundation length. For the second case we have faster convergence, and towards a lower limit.

We note that $n=0$ for the runs presented in this section. The singularity in the expression for bottom drag did not lead to unreasonable results. This is not always the case, as will be demonstrated subsequently.

\section{Run-up of the 1969 Gorringe Bank Tsunami.}

In this section we study the run-up and breaking of a tsunami at the coast of Portugal. The initial profile is extracted from a two-dimensional numerical solution of the Boussinesq equation, reported by Gjevik et al [1], at a simulation time where I expect that the dispersive effects may be neglected, and breaking may occur. The actual bottom topography is simplified substantially to consist of a linear slope, $5 \mathrm{~km}$ wide, connected to a flat bottom, $20 \mathrm{~m}$ deep, further out. The bottom topography and initial wave profile is shown in figure 3, upper panel. To enable a discussion of some principal aspects of this situation I have also made runs for a more shallow case, with depth $10 \mathrm{~m}$. The bottom drag is assumed to be given by $C_{D}=0.003$, and $n=0.5 m$, runs for $C_{D}=0$ and $n=0$ are also reported.

\subsection{Results.}

Converged numerical results showing the development of the surface profile during surf and run-up is demonstrated in figure 3 . We notice that no amplification takes place over the slope. The convergence for the shoreline particle is demonstrated in figure $4 \mathrm{a}$ ). Figure $4 \mathrm{~b}$ ) demonstrates that no convergence seems to take place during back-wash when the singularity in the expression for bottom stress is not removed. Of course the singularity is not physical, and we may conclude that an improved physical description of the thin film of fluid leads to better convergence. The same conclusion may be drawn from the results of the inclusion of the bottom friction term itself.

Figure $4 \mathrm{c}$ ) and $\mathrm{d}$ ) show similar results for the shallow case (maximum depth $10 \mathrm{~m}$ ). We notice that the curves have similar shapes as for the standard case. Now the waves have been damped during shoaling. 
a) Initial incident bore.

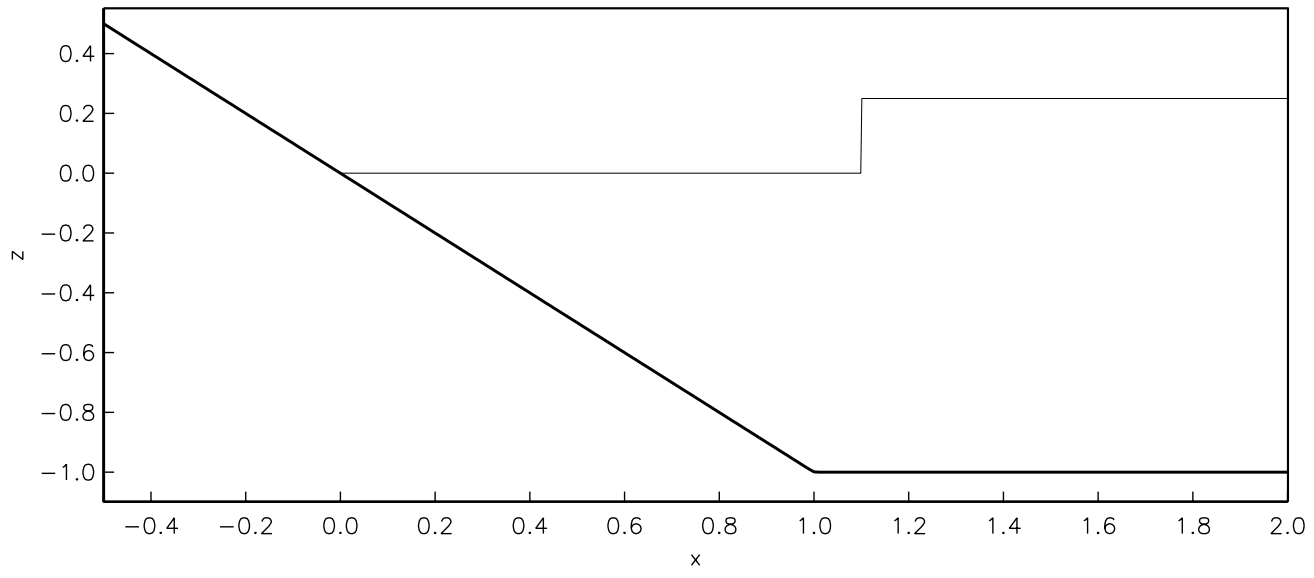

b) Surf.

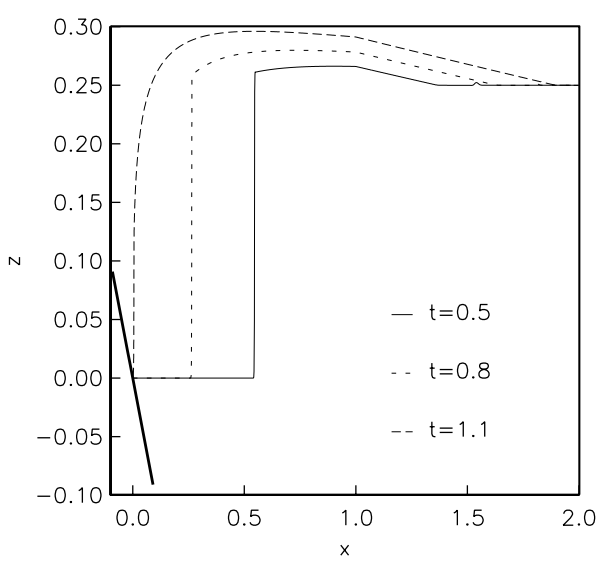

d) Back-wash.

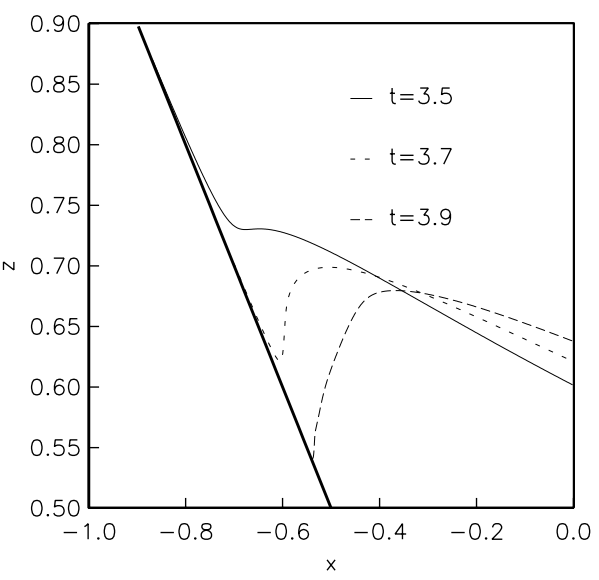

c) Run-up.

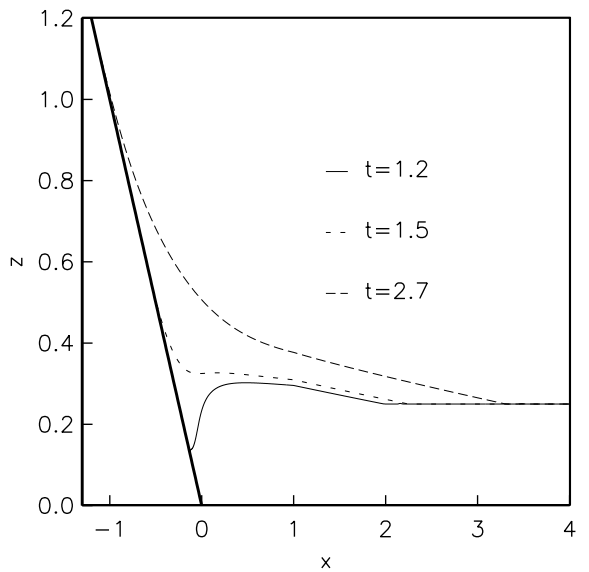

e) Reflection.

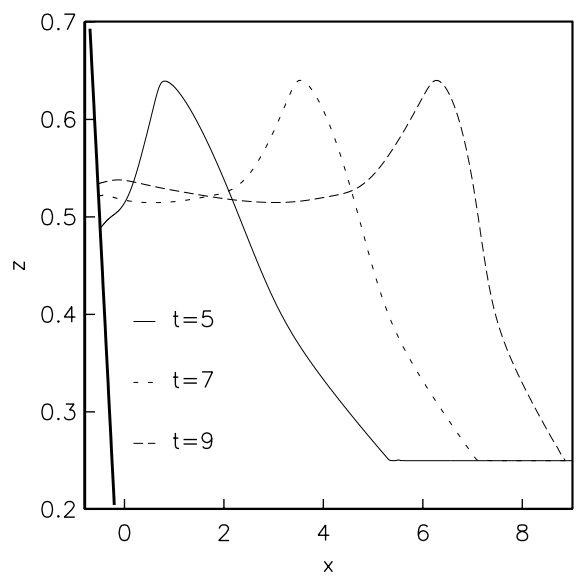

Figure 1: Surface profiles for bore run-up. Average initial grid size is $\Delta a$. 
a) Free tip elevation.

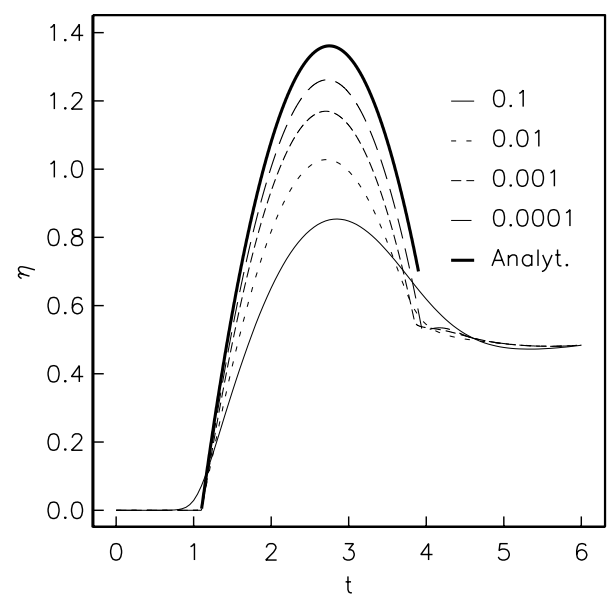

c) Free tip elevation, $\hat{C}_{D}=0.01$.

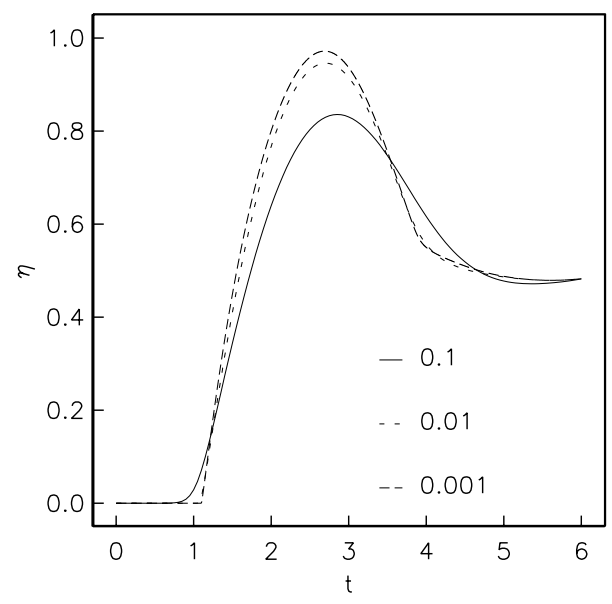

b) Modified run-up definition.

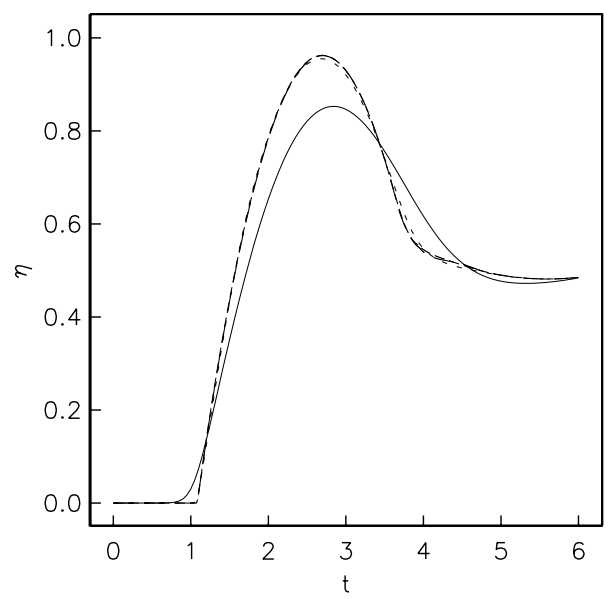

d) Free tip elevation, $\hat{C}_{D}=0.1$.

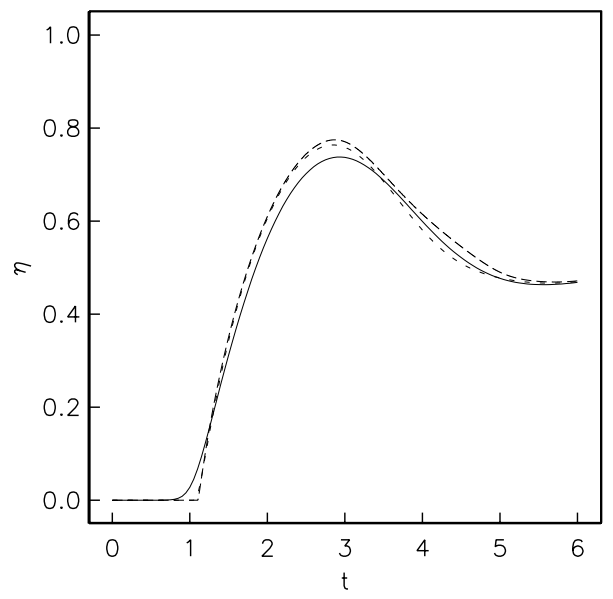

Figure 2: Grid refinement tests. Curve legends denote the average initial grid size. Right panels: curve legends given in left panels. 
Figure 4 e) shows that the bottom stress is vital to avoid slow convergence. As for the previous case we may speed up the convergence by redefine the inundation length, in figure $4 \mathrm{f}$ ) we track the position where the layer thickness is $0.25 \mathrm{~m}$.

\section{A simple model of the Tafjord event.}

A numerical study of the slide and tsunami event at Tafjord was given by Harbitz et. al, 93, with a linear and non-dispersive model. Probably both nonlinearity and dispersion should be considered. Here we study the effect of nonlinearity and wave breaking for a simplified fjord geometry, where the scales for the fjord and slide geometry is taken from Harbitz et. al. [2].

The bottom topography is assumed to be parabolic across and uniform along the fjord. The slide is $2 B_{s}$ wide, the frontal length is $L_{s}$ and the slide is uniform behind the front. The slide height is denoted $A$.

We now introduce a Cartesian coordinate system with $x$-axis across the fjord and $y$-axis along the beach where the slide is impinging. The slide center starts at $\left(x_{0}, 0\right)$ and travels a distance $R$, in a time $T_{R}$, along a line perpendicular to the initial shoreline. The bottom topography is then given by

$$
h(x, y, t)=h_{\max } 4 x(L-x) / L^{2}-\hat{h}\left(x-x_{r}(t), y\right),
$$

where $\hat{h}$ defines the shape of the slide and $x_{r}(t)=x_{0}+R\left(\sin \left(t \pi / 2 T_{R}\right)\right)$ is the position of the center of the slide. For $t>T_{R}$ the slide remains at rest. The slide body is described by the function

$$
\hat{h}(s, p)=A \cos ^{2}\left(\frac{s \pi}{2 L_{s}}\right) \cos ^{2}\left(\frac{p \pi}{2 B_{s}}\right),
$$

for $0<s<L_{s},-B_{s}<p<B_{s}$,

$$
\hat{h}(s, p)=A \cos ^{2}\left(\frac{p \pi}{2 B_{s}}\right),
$$

for $s<0,-B_{s}<p<B_{s}$,

and $\hat{h}=0$ elsewhere.

In the present case we have selected $A=75 \mathrm{~m}, L=1200 \mathrm{~m}, h_{\max }=180 \mathrm{~m}$, $L_{s}=B_{s}=133 m, x_{0}=-133 m, R=528 m, T_{R}=16.6 s$ and $C_{D}=0.003$.

\subsection{Results.}

The generation of waves due to the slide is demonstrated in figure 5 . We notice that a nearly semi-circular bore propagates away from the slide area and runs up on the opposite beach. Figure 6 shows grid refinement tests. We notice that the breaking is almost impossible to identify for the coarse grid. For the finest grid the results seem to have converged, except near the shoreline, this is consistent to the results from the previous two-dimensional cases. We also notice that the model seems to handle the situation with an obliquely incident 
Initial profile.

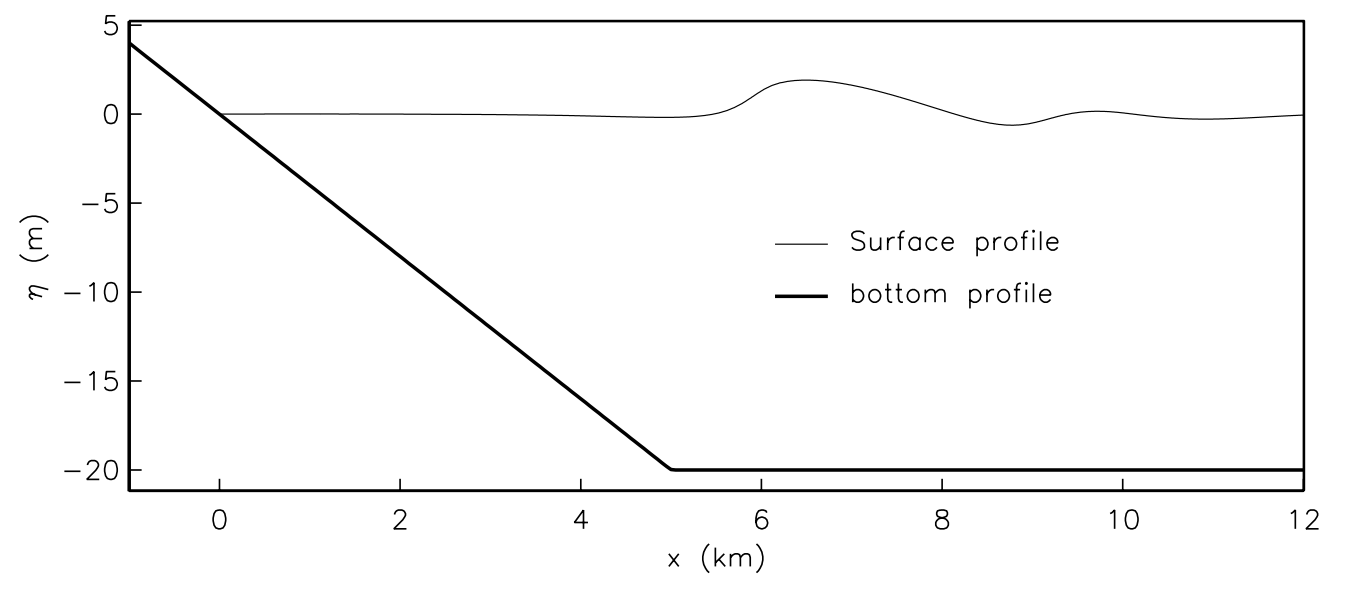

Surf.

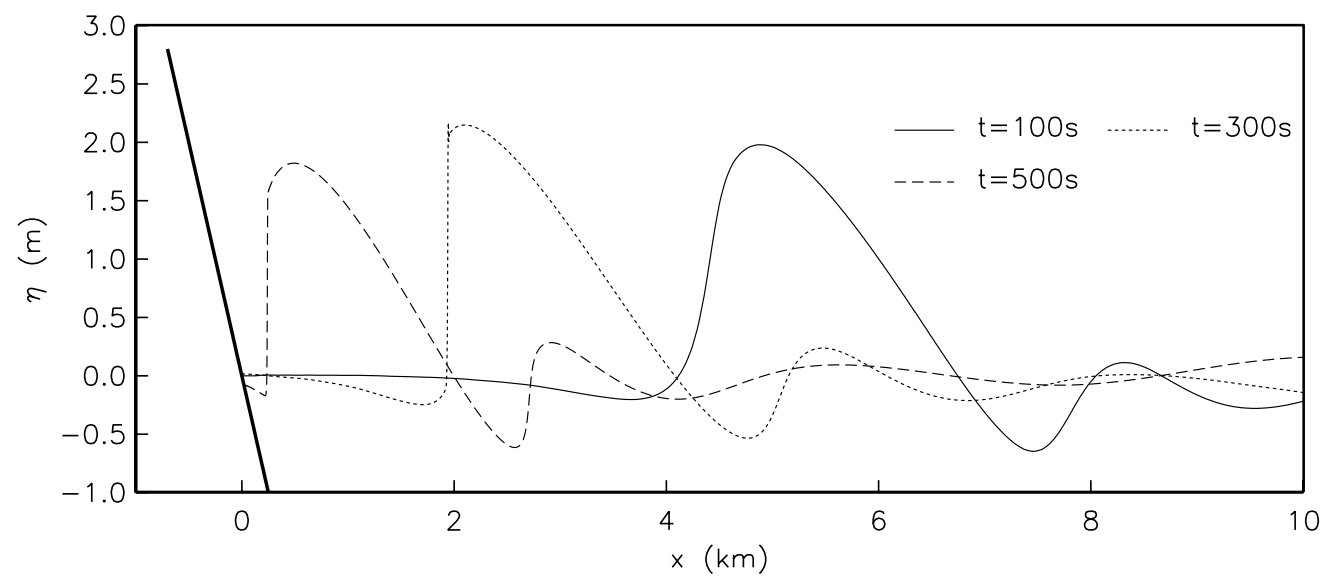

Run-up.

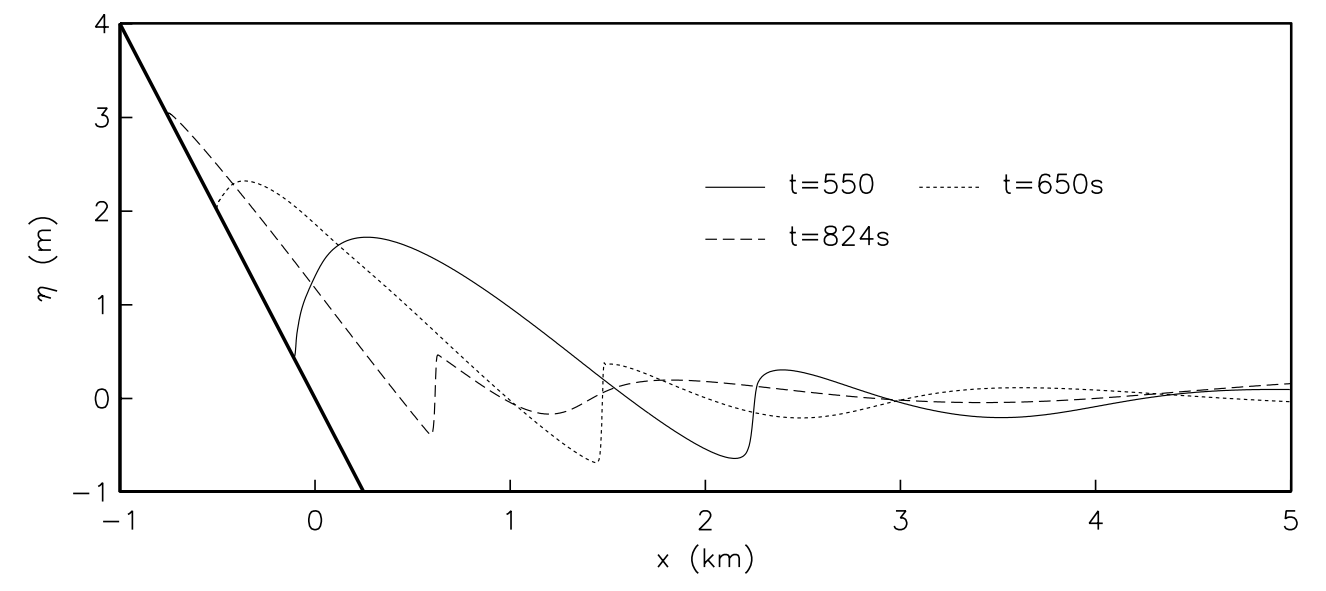

Figure 3: Surface profiles for Portuguese tsunami event. Average inital grid size is $5 \mathrm{~m}$. 
a) Normal case, $C_{D}=0.003,=0.5 \mathrm{~m}$.

b) As a), $n=0$.
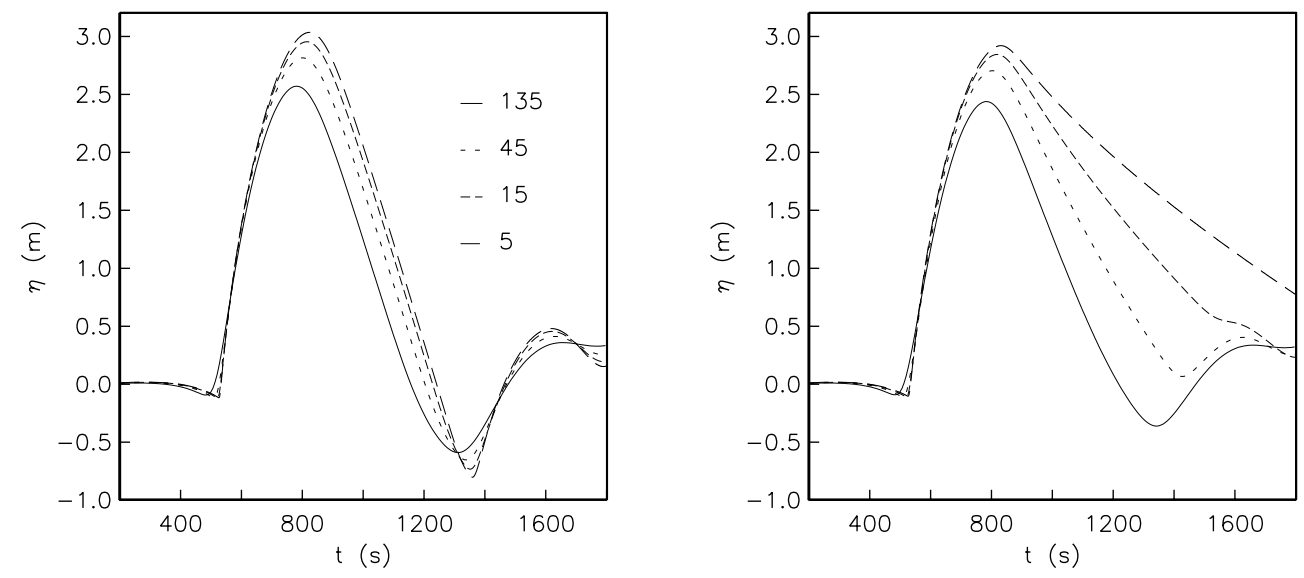

c) Shallow case, $C_{D}=0.003, n=0.5 \mathrm{~m}$.
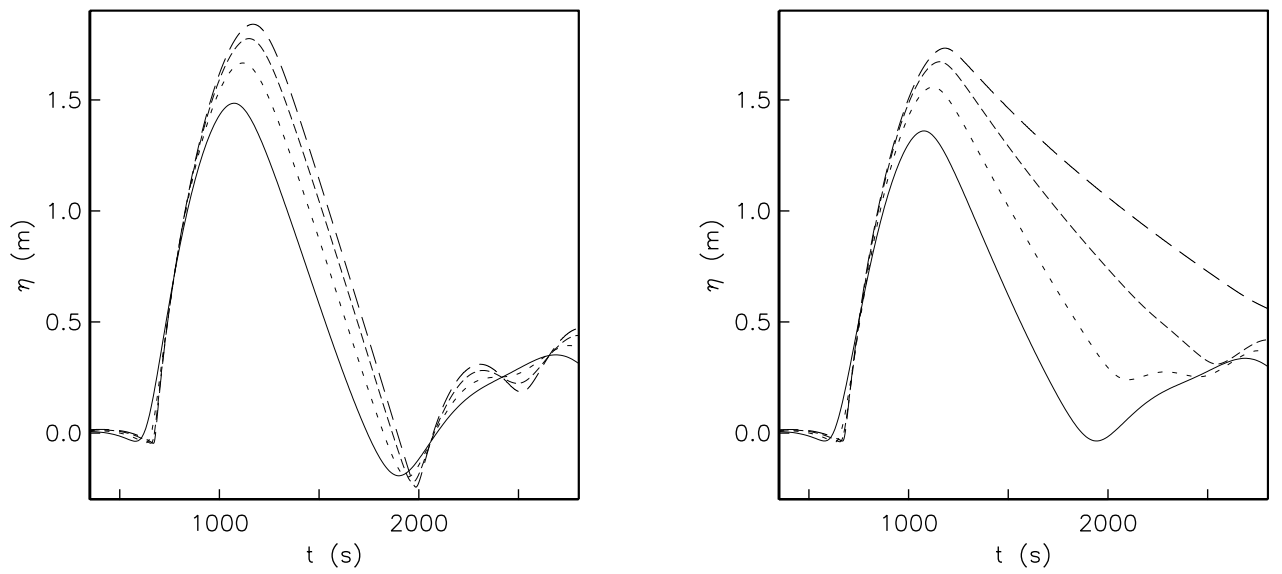

e) Normal case, $C_{D}=0$.

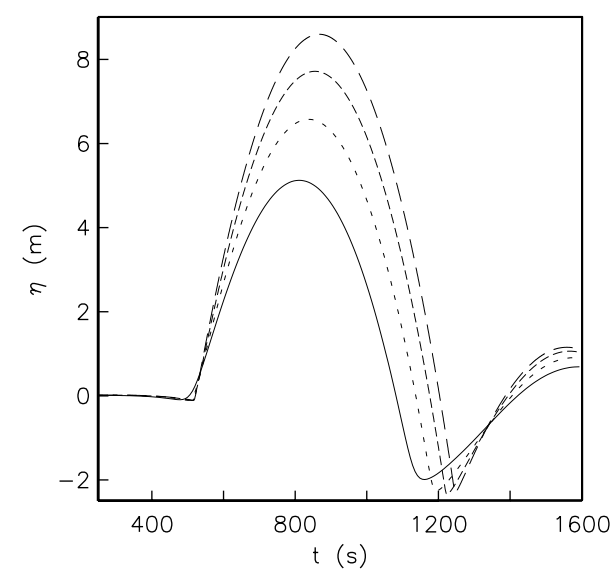

f) As e), mod. run-up def.

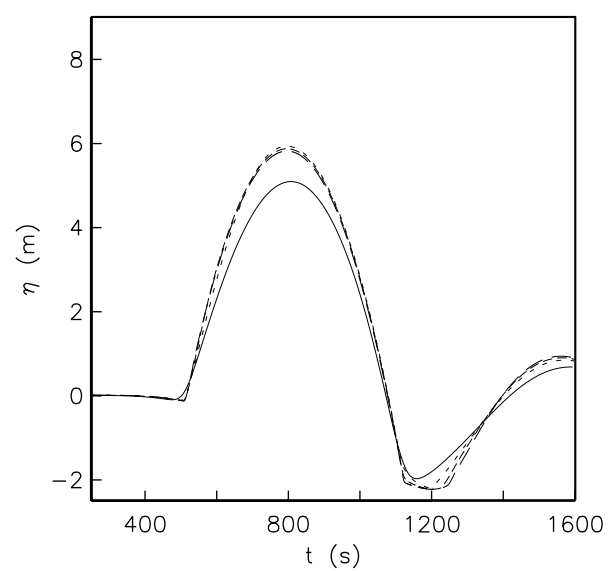

Figure 4: Free tip elevations, grid refinement tests. Curve legends given in upper left panel, denote the average initial grid size. 
bore on a shore. Finally, runs for $C_{D}=0$ showed that this parameter had no substantial impact on the present result. For very dense grid we expect the parameter to be important near the shore. The parameter would be more important for a more shallow fjord.

\section{Summary.}

A numerical model for three dimensional run-up of long waves has been generalized to include wave breaking. Comparison with analytical solutions showed convergence during grid refinement. The convergence was very slow, the reason why is the high grid density needed to resolve the details in the almost explosive bore development near the shoreline. The slow convergence is confined to a region near the free tip where the fluid layer is very thin during run-up and back-wash. An introduction of a bottom drag term, improving the physical description of the movement in this layer, speeded up the convergence substantially. This phenomenon was most pronounced for very shallow cases, where bottom drag must be accounted for anyway. It was necessary to remove the singularity in the expression for bottom drag to achieve fast convergence even for deep back-wash.

Many types of numerical diffusion, smoothening and filtering will impact wave breaking in a similar manner as the present method. For coarse grids the bores that are developed will, in general, be wide, and there is a good possibility that the breaking is not recognized. Figure 6, upper left panel, demonstrates this phenomenon. Grid refinements, linked to a reduction in numerical diffusion, will clear up the picture, as in the right panel.

\section{Acknowledgments.}

The present research has been partially financed by the Commission of the European Communities under the contract ENV4-U96-0297, as part of the international project GITEC-TWO, and by the Norwegian Research Council under contract EMSEFMK.300002. I am also grateful to Professor Geir Pedersen for interesting and fruitful discussions.

\section{References}

[1] Gjevik, B. et. al., Modeling tsunami from earthquake sources near Gorringe Bank southwest of Portugal, accepted in J. Geophysical research, 1997.

[2] Harbitz, C.B., Pedersen,G. and Gjevik, B., Numerical simulation of large water waves due to landslides. J. Hydraulic Engineering, 119(12), pp1325$1342,1993$.

[3] Hibberd, S. and Peregrine, D.H. Surf and run-up on a bore: a uniform bore. J. Fluid Mech. 95, pp323-345, 1979. 

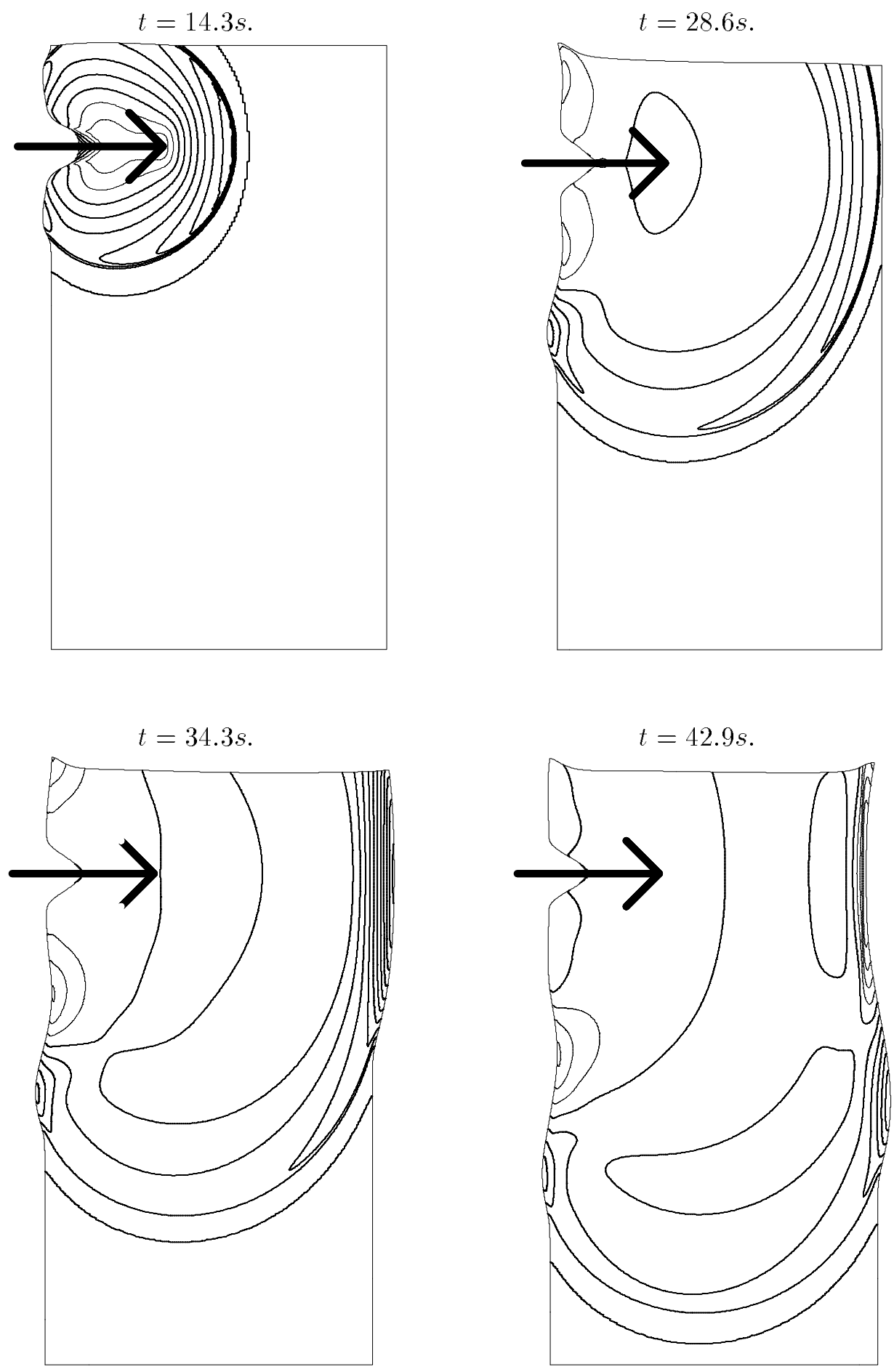

Figure 5: Time evolution of slide generated waves at Tafjord, 400 grid points across the fjord. Contour increments $5 \mathrm{~m}$, thick lines for elevation. 
50 grid points across fjord.

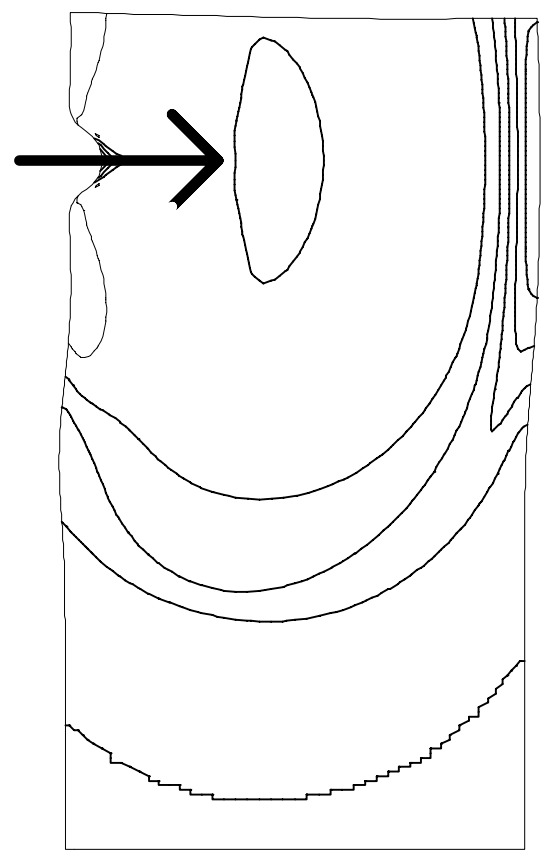

Fjord centre.

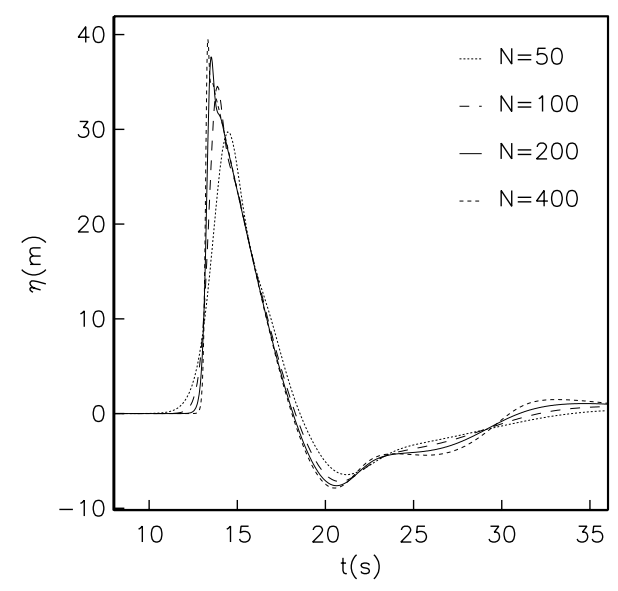

400 grid points across fjord.

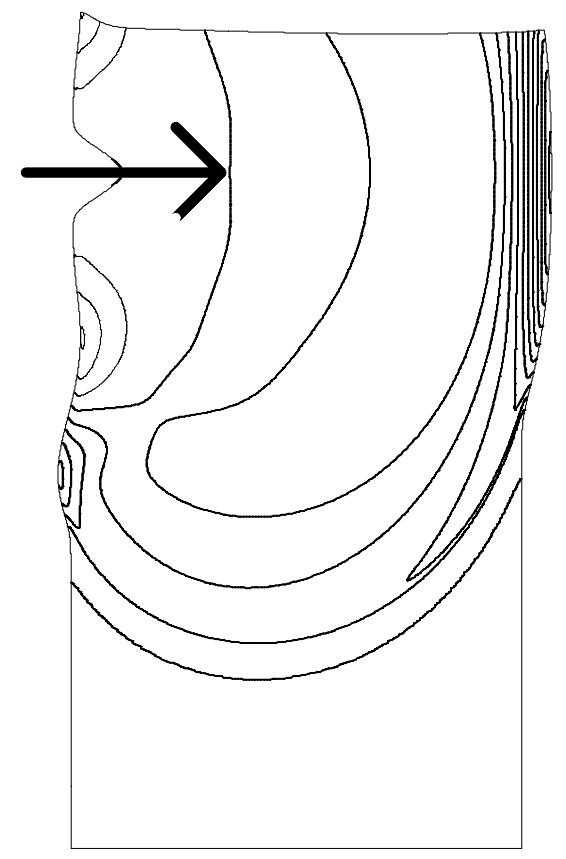

Oposite beach.

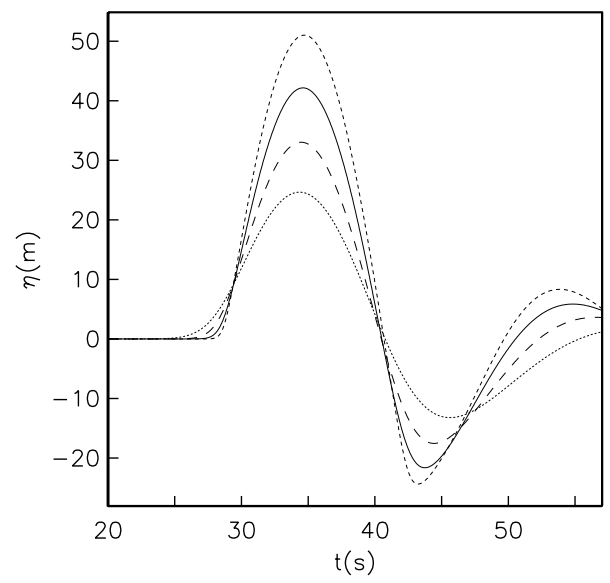

Figure 6: Grid refinement tests concerning the Tafjord case. Upper panels: $t=34.3 \mathrm{~s}$, contour increments $5 \mathrm{~m}$, thick lines for elevation. Lower panels: Time profiles of the surface elevation at fjord centre and oposite beach, curve legends given in left panel, $N$ donotes number of grid points across the fjord. 
[4] Johnsgard, H. and Pedersen, G., 1997, A numerical model for three dimensional run-up. Int. J. for Numerical Methods in Fluids. 24 pp913-931, 1997.

[5] Keller, H.B. et. al. Motion of a bore over a sloping beach. J. Fluid Mech. 7 pp302-316,1960.

[6] Peregrine.D.H., Equations for water waves and the approximations behind them. In: Waves on beaches. Ed. by R.E. Meyer, Academic Press, New York pp357-412, 1972.

[7] Shen, M.C. and Meyer, R.E. Climb of a bore on a beach 3: Run-up. J. Fluid Mech. 16 pp113-125,1963. 